\title{
Serum transglutaminase 3 antibodies correlate with age at celiac disease diagnosis
}

\section{Salmi, Teea T.}

2016-06

Salmi , T T , Kurppa , K, Hervonen , K, Laurila , K, Collin , P , Huhtala , H , Saavalainen , P , Sievanen , H , Reunala , T \& Kaukinen, K 2016 , ' Serum transglutaminase 3 antibodies correlate with age at celiac disease diagnosis ' , Digestive and Liver Disease , vol. 48 , no. 6 , pp. 632-637 . https://doi.org/10.1016/j.dld.2016.03.003

http://hdl.handle.net/10138/224014

https://doi.org/10.1016/j.dld.2016.03.003

publishedVersion

Downloaded from Helda, University of Helsinki institutional repository.

This is an electronic reprint of the original article.

This reprint may differ from the original in pagination and typographic detail.

Please cite the original version. 


\title{
Serum transglutaminase 3 antibodies correlate with age at celiac disease diagnosis
}

\author{
Teea T. Salmi ${ }^{a, b, *}$, Kalle Kurppa ${ }^{c}$, Kaisa Hervonen ${ }^{\text {a,b }}$, Kaija Laurila ${ }^{c}$, Pekka Collin ${ }^{\mathrm{d}}$, \\ Heini Huhtala ${ }^{\mathrm{e}}$, Päivi Saavalainen ${ }^{\mathrm{f}}$, Harri Sievänen ${ }^{\mathrm{g}}$, Timo Reunala ${ }^{\mathrm{a}, \mathrm{b}}$, Katri Kaukinen ${ }^{\mathrm{b}, \mathrm{h}}$ \\ a Department of Dermatology, Tampere University Hospital, Tampere, Finland \\ b School of Medicine, University of Tampere, Tampere, Finland \\ c Tampere Center for Child Health Research, University of Tampere and Tampere University Hospital, Tampere, Finland \\ d Department of Gastroenterology and Alimentary Tract Surgery, Tampere University Hospital, Tampere, Finland \\ e Tampere School of Health Sciences, University of Tampere, Tampere, Finland \\ ${ }^{\mathrm{f}}$ Department of Medical Genetics and Research Program for Molecular Medicine, Biomedicum Helsinki, University of Helsinki, Finland \\ g UKK Institute, Tampere, Finland \\ ${ }^{\mathrm{h}}$ Department of Internal Medicine, Tampere University Hospital, Tampere, Finland
}

\section{A R T I C L E I N F O}

\section{Article history:}

Received 19 January 2016

Accepted 2 March 2016

Available online 9 March 2016

\section{Keywords:}

Autoantibody

Dermatitis herpetiformis

Gluten-free diet

\begin{abstract}
A B S T R A C T
Background: Transglutaminase (TG)2 is the autoantigen in celiac disease, but also TG3 antibodies have been detected in the serum of celiac disease patients.

Aims: To investigate the correlations between serum TG3 antibodies and clinical and histological manifestations of celiac disease and to assess gluten-dependency of TG3 antibodies.

Methods: Correlations between serum TG3 antibody levels measured from 119 adults and children with untreated coeliac disease and the demographic data, clinical symptoms, celiac antibodies, histological data and results of laboratory tests and bone mineral densities were tested. TG3 antibodies were reinvestigated in 97 celiac disease patients after 12 months on a gluten-free diet (GFD).

Results: TG3 antibody titers were shown to correlate with the age at celiac disease diagnosis. Further, negative correlation with TG3 antibodies and intestinal $\gamma \delta+$ cells at diagnosis and on GFD was detected. Correlations were not detected with the clinical manifestation of celiac disease, TG2 or endomysial autoantibodies, laboratory values, severity of mucosal villous atrophy, associated diseases or complications. TG3 antibody titers decreased on GFD in $56 \%$ of the TG3 antibody positive patients.

Conclusion: Serum TG3 antibody positivity in celiac disease increases as the diagnostic age rises. TG3 antibodies did not show similar gluten-dependency as TG2 antibodies.
\end{abstract}

(C) 2016 Editrice Gastroenterologica Italiana S.r.l. Published by Elsevier Ltd. All rights reserved.

\section{Introduction}

Celiac disease is an autoimmune mediated disorder induced by gluten in genetically susceptible individuals. The clinical appearance of celiac disease is typically a chronic gastrointestinal disorder, in which mucosal villous atrophy and crypt hyperplasia are detectable in small bowel biopsy specimens. Transglutaminase (TG)2 has been identified as the major autoantigen in celiac disease [1]. TG2 antibodies have been proven to be highly accurate in celiac disease diagnostics, and there is also solid evidence of

\footnotetext{
* Corresponding author at: Department of Dermatology, Tampere University Hospital, P.O. Box 2000, 33521 Tampere, Finland. Tel.: +358 40 5863818; fax: +358331165654.

E-mail address: teea.salmi@uta.fi (T.T. Salmi).
}

gluten-dependency of these antibodies. Altogether nine distinct transglutaminases exist in a wide variety of cell types in humans, and even though their functions generally involve tissue assembly or repair, it is nowadays recognized that transglutaminases are inappropriately activated in a variety of pathologies [2]. Dermatitis herpetiformis $(\mathrm{DH})$ is a cutaneous manifestation of celiac disease causing itching and blistering rash [3], but DH patients further evince celiac-type small bowel mucosal villous atrophy or inflammation [4,5]. In DH pathognomonic granular IgA deposits can be detected by direct immunofluorescence (IF) in the papillary dermis, and it has previously been shown that this $\operatorname{IgA}$ is directed against TG3 [6]. TG3 is an enzyme expressed in the epidermis and it is closely related to, but not identical to, TG2 [7]. Vast majority of DH patients, similarly as celiac disease patients, present circulating and intestinal autoantibodies against TG2 at the time of the diagnosis $[8,9]$. In addition, TG3 autoantibodies have been identified in the 
serum of not only untreated DH but also of celiac disease patients [6,10-12]. However, thus far it is obscure what is the role of TG3 antibodies in celiac disease, and whether discrepant autoantibody response and TG3 antibodies might have a crucial bearing in the development of these two distinct manifestations of celiac disease.

This study aimed to elucidate the significance of TG3 antibodies in celiac disease. We investigated whether TG3 antibodies correlate with the age at diagnosis, severity of clinical symptoms or histological inflammation or damage in celiac disease. Further, gluten-dependency of TG3 antibodies was evaluated with serial serum samples of celiac disease patients adhering to a gluten-free diet (GFD) treatment.

\section{Methods}

\subsection{Study patients}

Serum TG3 antibodies were investigated from 119 adults and children diagnosed with celiac disease at the Tampere University Hospital, Finland. Children with a celiac disease suspicion were enrolled prospectively. All study patients had biopsy proven celiac disease. Patients with DH were not included in this study, but the presence of other skin symptoms was collected. Primary reason for celiac disease suspicion, the duration of symptoms before diagnosis, the presence of celiac disease associated disorders and family history of celiac disease or DH were recorded. Serum TG3 antibodies were reinvestigated from 97 celiac disease patients after they had adhered to a GFD for 12 months, and at that time also clinical response and the strictness of GFD were documented. The strictness of GFD was classified as strict when no dietary lapses existed, partial when dietary lapses existed or no adherence to GFD. The study protocols and celiac children recruitment were approved by the Ethics Committee of Tampere University Hospital District. All study participants or their parents gave written informed consent.

\subsection{Celiac antibodies, laboratory parameters and celiac disease genetics}

TG3 antibodies were measured with IgA anti-TG3 ELISA kits (Immunodiagnostik, Bensheim, Germany) and TG2 antibodies with IgA anti-TG2 ELISA kits (Celikey; Phadia, GmbH, Freiburg, Germany). In addition, antibodies to deamidated gliadin (DGP) were measured with anti-DPG ELISA kits (IgA + IgG antibodies, QuantaLite Celiac DGP Screen; INOVA Diagnostics Inc., San Diego, CA). The TG3 antibody kit provides an ELISA plate coated with recombinant TG3 from human source (C-terminal antigen - MW $77 \mathrm{kDa}$, Locus ID 7053). The sera were diluted according to manufacturer's instructions by 1:250 when TG3 antibodies were analyzed and by 1:100 for TG2 antibody and DPG antibody analyzes. The TG3 antibodies were measured in double, and TG2 and DPG antibodies in single runs. A positive and negative control from manufacturer were included in each run. TG3 values $\geq 16 \mathrm{AU} / \mathrm{ml}$ were considered as positive according to the manufacturer's instructions, and the cut-off value of TG2 antibodies was $3.0 \mathrm{AU} / \mathrm{ml}$ and of DGP antibodies $20 \mathrm{AU} / \mathrm{ml}$.

Serum IgA class endomysial antibodies (EmA) were determined by an indirect IF method using human umbilical cord as substrate as previously described in detail [13]. A titer of $1: \geq 5$ for EmA was considered positive.

The following laboratory values were measured by standard laboratory methods: blood hemoglobin, serum iron, erythrocyte folic acid, serum vitamin B12 and serum parathyroid hormone levels. Hemoglobin values of children $<16$ years of age and in patients $\geq 16$ years of age were further analyzed separately due to different reference values among children and adults.
The presence of celiac disease-associated human leukocyte antigen (HLA) DQB1*02 and DQB1*0302 alleles (DQ2 and DQ8) was investigated by using either the DELFIA Celiac Disease Hybridization Assay (PerkinElmer Life and Analytical Sciences, Wallac Oy, Turku, Finland) or the SSP DQBI low-resolution kit (Olerup SSP $\mathrm{AB}$, Saltsjöbaden, Sweden/Qiagen Vetriebs GmbH, Vienna, Austria) according to the manufacturer's instructions.

\subsection{Small bowel biopsy}

During esophago-gastroduodenoscopy a minimum of six forceps biopsy specimens were taken from the duodenum. Three to five of these were processed, stained with hematoxylin and eosin and studied under light microscopy. The density of intraepithelial lymphocytes (IELs) and villous height-crypt depth ratios ( $\mathrm{Vh} / \mathrm{CrD}$ ) were determined from several well-oriented biopsy samples from multiple sites as previously described [14]. IELs were counted per 100 enterocytes, and $\mathrm{Vh} / \mathrm{CrD}>2$ was considered normal. In addition, the mucosal specimens were graded independently according to the Marsh Classification as follows: Marsh I-II denoted normal villi together with intraepithelial lymphocytosis and normal (Marsh I) or elongated crypts (Marsh II), and Marsh III destructive lesion with villous atrophy and crypt hyperplasia [15].

Two to three small bowel biopsy specimens were freshly embedded in optimal cutting temperature compound (OCT, TissueTec, Miles Inc, Elkhart, IN), snap-frozen in liquid nitrogen and stored at $-70^{\circ} \mathrm{C}$. Immunohistochemical stainings were carried out on 5$\mu \mathrm{m}$-thick frozen sections. CD3+ IELs were stained with monoclonal antibody Leu-4 (Becton Dickinson, San Jose, CA) and $\gamma \delta+$ IELs with TCR $\gamma$ antibody (Endogen, Woburn, MA). Positive IELs were counted with a $100 \times$ flat field light microscope objective throughout the surface epithelium as previously described [5], and IEL density was expressed as cells per millimeter of epithelium.

Small bowel mucosal TG2-specific IgA deposits were investigated from frozen small bowel sections as previously described [16]. Altogether six unfixed, 5 - $\mu \mathrm{m}$-thick sections from frozen small bowel specimens were processed, three for investigation of IgA deposits and three for double-color labeling for both IgA and TG2. IgA was detected by direct IF as described by Korponay-Szabo et al. [16]. The occurrence of the IgA deposits was graded semiquantitatively according to their intensity along the basement membrane in the villous-crypt area as follows: negative, weak $(+)$, moderate $(++)$, and strong positive $(+++)$. In cases where the intensity was patchy, it was graded from different areas and the mean value was given. Furthermore, sections were double-stained for human IgA (green) and for TG2 (red) to confirm that coeliac-type IgA deposits co-localized with TG2.

\subsection{Body mass index (BMI), bone mineral density (BMD) and study questionnaires}

BMI was calculated as weight per square of height $\left(\mathrm{kg} / \mathrm{m}^{2}\right)$, and BMI values of children $<16$ years of age and in patients $\geq 16$ years of age were further analyzed separately. BMD was measured in the lumbar spine and left femoral neck by dual-energy X-ray absorptiometry according to our standard procedures [17]. BMD values were expressed as standard deviation scores that compare individual BMD values with those of sex-matched healthy young adults ( $T$-score); $T$-score above -1.0 indicated normal BMD, between -1.0 and -2.4 osteopenia and values -2.5 or below osteoporosis.

Gastrointestinal symptoms and health-related quality of life at the time of the diagnosis were measured with the Gastrointestinal Symptom Rating Scale (GSRS)- and Psychological General WellBeing (PGWB) questionnaires, which are structured and validated questionnaires widely applied in celiac disease studies [18,19]. GSRS consists of 15-items and uses a seven-grade Likert scale for 
Table 1

Demographic and clinical data of all celiac disease (CD) patients and serum transglutaminase (TG) 3 antibody positive and -negative CD patients at diagnosis.

\begin{tabular}{|c|c|c|c|}
\hline & All CD patients $(n=119)$ & $\begin{array}{l}\text { TG3 antibody positive } \\
\text { CD patients }(n=33)\end{array}$ & $\begin{array}{l}\text { TG3 antibody negative } \\
\text { CD patients }(n=86)\end{array}$ \\
\hline Females; $n(\%)$ & $83 / 119(70)$ & $23 / 33(70)$ & $60 / 86(70)$ \\
\hline Age; median (range) & $36(3-71)$ & $47(13-69)^{*}$ & $25(3-71)^{*}$ \\
\hline \multicolumn{4}{|l|}{ Primary reason for $\mathrm{CD}$ suspicion } \\
\hline Abdominal $^{\mathrm{a}}$ & $59 / 119(50)$ & $19 / 33(58)$ & $40 / 86(46)$ \\
\hline Malabsorption $^{\mathrm{b}}$ & $9 / 119(8)$ & $4 / 33(12)$ & $5 / 86(6)$ \\
\hline Extraintestinal symptoms ${ }^{c}$ & $16 / 119(13)$ & $4 / 33(12)$ & $12 / 86(14)$ \\
\hline Screening at-risk groups ${ }^{\mathrm{d}}$ & $35 / 119(29)$ & $6 / 33(18)$ & $29 / 86(34)$ \\
\hline Duration of symptoms before CD diagnosis, years; median (range) & $3(0-57)$ & $5(0-50)$ & $2(0-57)$ \\
\hline CD associated diseases ${ }^{e}$ & $25 / 79(32)$ & $13 / 32(41)$ & $12 / 47(26)$ \\
\hline Family history of $\mathrm{CD} ; n(\%)$ & $66 / 118(56)$ & $16 / 33(48)$ & $50 / 85(59)$ \\
\hline
\end{tabular}

${ }^{*} P<0.001$ when TG3 antibody positive and -negative patients were compared.

a Abdominal pain or distension, diarrhea, constipation, flatulence.

b Anemia.

c Skin symptoms (other than dermatitis herpetiformis), joint symptoms, tongue pain, osteoporosis, infertility.

d Family history of celiac disease.

e Autoimmune thyroid disease, type I Diabetes, Sjögren's disease, IgA nephropathy, epilepsy, atrophic gastritis.

each item, and it evaluates gastrointestinal symptoms in five different subgroups: diarrhea, ingestion, constipation, abdominal pain and reflux. Higher scores indicate more severe gastrointestinal symptoms [20]. PGWB is a 22-item questionnaire using a six-grade Likert scale, and it is used to assess the health-related quality of life and well-being. The total score of PGWB ranges therefore between 22 and 132 points, higher score indicating better quality of life [18].

\subsection{Statistics}

Quantitative data were expressed as the number of subjects and percentages, or as medians and ranges. To assess statistical differences between antibody positive and negative patients, Pearson Chi-Square test of Fisher's exact test was used, as appropriate. Spearman's correlation coefficient was applied to assess correlations between different variables. A $P$ value less than 0.05 was considered statistically significant. The data were analyzed with the SPSS Statistics 20 software package (International Business Machines Corp., New York, USA).

\section{Results}

Eighty-three (70\%) out of the 119 untreated celiac disease patients were females and 36 (30\%) males (Table 1). The median age at diagnosis was 36 (range 3-71) years, and 37 patients (31\%) were under 16 years of age at the time of the diagnosis. Thirty-three (28\%) of all 119 untreated celiac disease patients had TG3 antibody titers above the cut-off level. TG3 antibody positive patients were statistically significantly older than antibody negative (Table 1 ) and also TG3 antibody titers were shown to correlate with the age at diagnosis $(r=0.461, P<0.001)$. Only one patient $<16$ years of age had positive TG3 antibodies. Gender distribution was similar between TG3 antibody positive and -negative patients (Table 1) and primary reasons for celiac disease suspicion or the duration of symptoms before diagnosis did not differ between antibody positive and negative patients (Table 1 ). The presence of skin symptoms other than DH was present in $12 \%$ of both TG 3 antibody positive and-negative patients. HLA genotypes did not differ between TG3 antibody positive and negative patients; $87 \%$ and $85 \%$ of the patients were HLA DQ2 positive, $3 \%$ and $4 \%$ HLA DQ8 positive, and $10 \%$ and $11 \%$ HLA DQ2 and DQ8 positive, respectively. A higher percentage of TG3 antibody positive patients had celiac disease associated disorders such as thyroid disorders, Sjögren's disease or type I diabetes, but the difference was not statistically significant (Table 1 ).

The severity of mucosal lesion measured with Marsh Classification at the time of the diagnosis did not differ between TG3 antibody positive and -negative patients $(P=0.390)$, and no correlation was detected between $\mathrm{Vh} / \mathrm{CrD}$ and TG3 antibody titers (Table 2). However, there was a weak negative correlation between TG3 antibody titers at diagnosis and $\mathrm{Vh} / \mathrm{CrD}$ measured after 12 months of GFD (Table 2). A weak negative correlation was detected between TG3 antibodies and $\gamma \delta+$ IEL densities at diagnosis and the negative correlation was stronger with TG3 antibodies and $\gamma \delta+$ IELs on GFD (Table 2). The densities of IELs or CD3+ IELs did not correlate with TG3 antibody titers.

TG3 antibodies at diagnosis showed no correlation with TG2 antibody titers, EmA titers (Table 2), or DGP antibody titers $(r=-0.105, P=0.490)$. Significant correlations with TG3 antibodies and measured laboratory values, BMI or BMD measurements were not detected either (Table 3). PGWB total scores or GSRS total (Table 3) or subdimension scores (data not shown) did not show correlations with TG3 antibodies.

TG2 antibodies were positive in 95\% (112 out of 118) of the patients at the time of the diagnosis. TG2 antibody titers at diagnosis were found to have a positive correlation with EmA titers $(r=0.800, P<0.001)$, DPG antibodies $(r=0.606, P<0.001)$,

Table 2

Correlations between serum transglutaminase (TG) 3 antibody titers at the time of celiac disease (CD) diagnosis and celiac disease related serological and histological data.

\begin{tabular}{|c|c|c|c|}
\hline & $\begin{array}{l}\text { Number of } \\
\text { studied CD } \\
\text { patients }\end{array}$ & $\begin{array}{l}\text { Correlation } \\
\text { coefficient with } \\
\text { serum TG3 titers }\end{array}$ & $P$ value \\
\hline \multicolumn{4}{|l|}{ EmA titer } \\
\hline At diagnosis & 118 & -0.156 & 0.091 \\
\hline 12 months on GFD & 71 & -0.145 & 0.227 \\
\hline \multicolumn{4}{|l|}{ TG2 antibody titer } \\
\hline At diagnosis & 118 & -0.034 & 0.714 \\
\hline 12 months on GFD & 71 & 0.014 & 0.905 \\
\hline \multicolumn{4}{|l|}{$\mathrm{Vh} / \mathrm{CrD}$} \\
\hline At diagnosis & 79 & 0.136 & 0.231 \\
\hline 12 months on GFD & 56 & -0.260 & 0.053 \\
\hline \multicolumn{4}{|l|}{ Density of IELs } \\
\hline At diagnosis & 79 & -0.012 & 0.919 \\
\hline 12 months on GFD & 14 & -0.016 & 0.958 \\
\hline \multicolumn{4}{|l|}{ Density of CD3+ IELs } \\
\hline At diagnosis & 77 & -0.181 & 0.114 \\
\hline 12 months on GFD & 22 & -0.326 & 0.138 \\
\hline \multicolumn{4}{|l|}{ Density of $\gamma \delta+$ IELs } \\
\hline At diagnosis & 75 & -0.245 & 0.034 \\
\hline 12 months on GFD & 35 & -0.581 & $<0.001$ \\
\hline $\begin{array}{l}\text { Intensity of intestinal IgA } \\
\text { deposits at diagnosis }\end{array}$ & 72 & -0.094 & 0.433 \\
\hline
\end{tabular}

EmA, endomysial antibodies; GFD, gluten-free diet; $\mathrm{Vh} / \mathrm{CrD}$, villous height crypt depth ratio; IELs, intraepithelial lymphocytes. 
Table 3

Correlations between serum transglutaminase (TG)3 antibody results and laboratory values, bone mineral densities and results of Psychological General Well-Being (PGWB) and Gastrointestinal Symptom Rating Scale (GSRS) -questionnaires at the time of celiac disease (CD) diagnosis.

\begin{tabular}{llll}
\hline & $\begin{array}{l}\text { Number of } \\
\text { studied CD } \\
\text { patients }\end{array}$ & $\begin{array}{l}\text { Correlation } \\
\text { coefficient with } \\
\text { serum TG3 titers }\end{array}$ & P value \\
\hline Body mass index & 85 & 0.241 & $0.027^{\mathrm{a}}$ \\
Hemoglobin & 96 & 0.064 & $0.536^{\mathrm{a}}$ \\
Vitamin B12 & 76 & 0.173 & 0.134 \\
Erythrocyte folic acid & 76 & -0.022 & 0.849 \\
Total iron & 85 & -0.039 & 0.720 \\
Parathyroid hormone level & 45 & -0.245 & 0.105 \\
Lumbar T-score & 70 & -0.084 & 0.487 \\
Femur T-score & 70 & 0.112 & 0.356 \\
PGWB total score & 63 & 0.093 & 0.469 \\
GSRS total score & 67 & -0.184 & 0.136 \\
\hline
\end{tabular}

a Statistically significant $P$ value was not detected when patients $<16$ years of age and patients $\geq 16$ years of age where analyzed separately.

densities of CD3+ IELs $(r=0.344, P=0.002)$, the intensity of intestinal IgA deposits $(r=0.390, P=0.001)$, and a weak correlation with $\gamma \delta+$ IELs $(r=0.273, P=0.019)$. A weak negative correlation was found between TG2 antibody titers and $\mathrm{Vh} / \mathrm{CrD}$ at the time of the diagnosis $(r=-0.231, P=0.041)$.

After one year of GFD more specific dietary adherence data were available in 79 adult patients, and $95 \%$ were on a strict diet and the remaining patients on a partial GFD. Clinical response, i.e. alleviance of celiac disease related symptoms, was documented in all but one patient. TG3 antibody results on GFD were available in 97 celiac disease patients, and antibody titers had remained at the same level or increased in $66 \%$ and decreased in $34 \%$ of the patients (Fig. 1a). TG3 antibody titers decreased along with GFD in 56\% of the patients with TG3 antibody titers above the cut-off level at diagnosis and in $23 \%$ of the patients with values below the cut-off $(P=0.001)$. TG2 antibody titers decreased on GFD in all but one (70/71) patient with available data (Fig. 1b), and this patient was TG2 antibody negative at diagnosis.

\section{Discussion}

The major outcome of this study was that TG3 antibody titers increase as the age at celiac disease diagnosis rises. Also a weak negative correlation was detected between TG3 antibodies and densities of $\gamma \delta+$ IELs at diagnosis, and a significant negative correlation with $\gamma \delta+$ IELs on GFD. Further, a weak negative correlation was seen in TG3 antibodies and $\mathrm{Vh} / \mathrm{CrD}$ on GFD suggesting that celiac disease patients with higher TG3 antibodies at diagnosis show poorer mucosal recovery on GFD.

TG3 is known to be the autoantigen of $\mathrm{DH}$, and $\mathrm{DH}$ patients invariably express IgA response directed against TG3 in the papillary dermis [6]. DH is a cutaneous manifestation of celiac disease affecting approximately 1 out of 8 celiac disease patients nowadays [21]. Small bowel mucosal alterations are similar in celiac disease and $\mathrm{DH}$, both conditions are gluten-dependent, and further, $\mathrm{DH}$ and celiac disease share similar genetic background and can occur in the same families or even in identical twins [22]. However, fundamental reasons for these two different expressions of celiac disease are obscure. Our hypothesis has been for long that DH is a late manifestation of undetected or neglected celiac disease. We have earlier reported that approximately $4 \%$ of newly detected $\mathrm{DH}$ patients have a previous celiac disease diagnosis, and this phenotype change was often connected to poor dietary compliance [23]. We have further shown that simultaneously as the incidence of celiac disease has increased [24] the incidence of DH has decreased [21], probably to a large extent caused by better diagnostic accuracy of celiac disease.
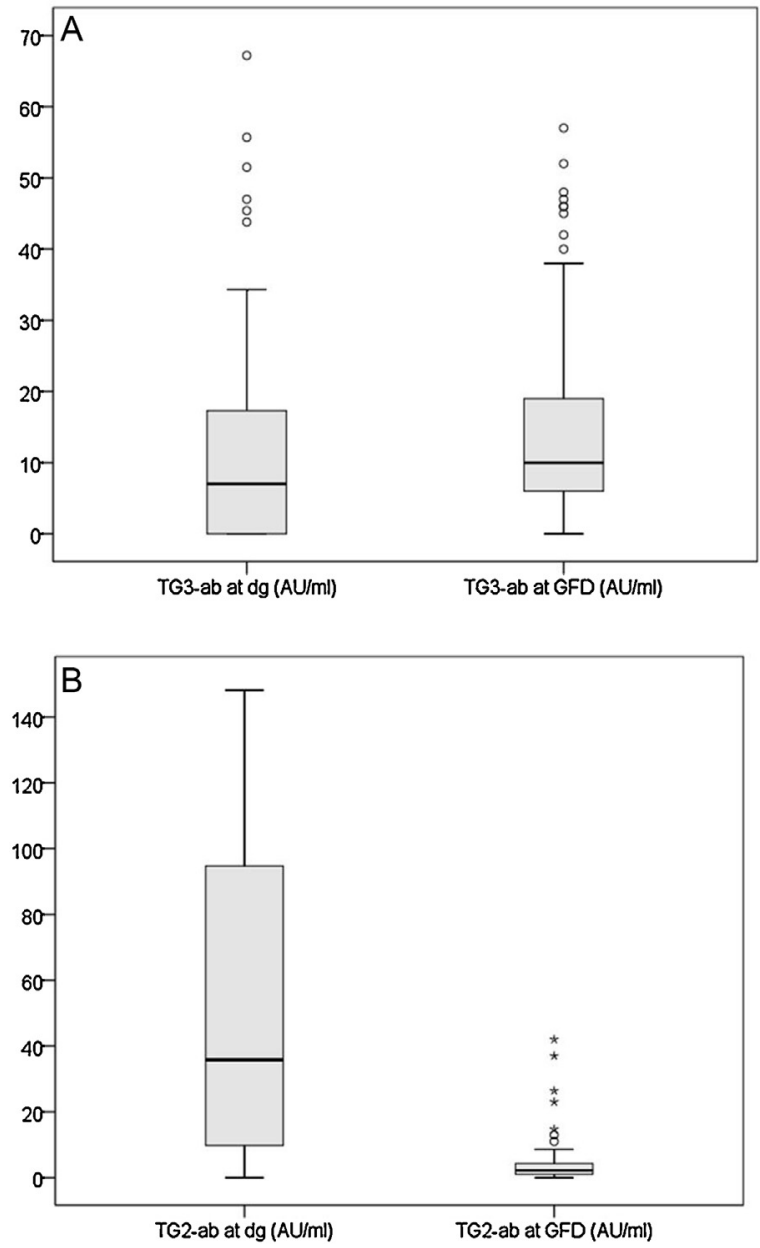

Fig. 1. (a) Median values, lower and upper interquartiles and ranges of serum transglutaminase 3 antibodies (TG3-ab) in 119 celiac disease patients at diagnosis (at dg) and in 97 celiac disease patients after 12 months of gluten-free dietary treatment (at GFD). One celiac disease patient had TG3-ab titer of 189 at the time of the diagnosis, which is not shown in the figure. (b) Median values, lower and upper interquartiles and ranges of serum transglutaminase 2 antibodies (TG2-ab) in 118 celiac disease patients at diagnosis (at dg) and in 71 celiac disease patients after 12 months of gluten-free dietary treatment (at GFD). One celiac disease patient had TG2-ab titer of 251 at the time of the diagnosis, which is not shown in the figure

The occurrence of circulating antibodies to TG3 might thus be a result of epitope spreading from an autoantibody response initially directed against TG2 in celiac disease, and this epitope spreading could be a phenomenon connected to a prolonged gliadin provocation. It has further been speculated that the TG3 antibody response develops in those celiac disease patients who will go on to acquire $\mathrm{DH}$. The results of this study support this speculation: TG3 antibody positivity was very rare among children diagnosed with celiac disease and our results were parallel to previous reports by Hull et al. [12] and Jaskowski et al. [25]. Only one paediatric patient in our current series had elevated levels of TG3 antibodies. Similarly DH is rarely diagnosed during childhood: we have previously shown that in Finland only $4 \%$ of patients contract DH before adulthood [21]. Further, even though in this study statistically significant difference was not detected in primary symptoms suggesting celiac disease, a higher percentage of TG3 antibody negative patients compared to -positive patients were diagnosed due to screening at-risk groups. It could be that screen-detected patients are diagnosed earlier, and among these patients the occurrence of epitope spreading is not yet as frequent. Whether serum TG3 antibody measurement might have value in identifying celiac disease patients with an increased risk of developing celiac disease related skin 
symptoms is an intriguing issue, but this remains to be elucidated in the future.

Previous data about serum TG3 antibodies in celiac disease and DH have been conflicting in several aspects. Some, but not all, of the discrepancies in earlier studies might be explainable by the usage of different methodologies or variable cut-off values. In the majority of the studies TG3 antibody titers have been higher among DH than in celiac disease patients $[10,12,26-28]$ and TG3 antibody positivity more prevalent in DH compared to celiac disease $[10,11,25,28]$, as expected. However, majority of studies focusing on serum TG3 antibodies have been centered around DH patients, and more limited data are available about TG3 antibodies in celiac disease. Correlations between TG3 and TG2 antibodies in celiac disease have been detected in some [27,29] but not in all studies [11]. Borroni et al. [19] published a study showing correlation between TG3 antibody titers and weight loss; no correlation was detected between TG3 antibodies and villous atrophy, age at celiac disease diagnosis, associated diseases or the presence of complications. Apart from the age at celiac disease diagnosis as a significant factor, we could not find correlations between clinical manifestations of celiac disease, associated diseases or the presence of complications either, and no correlation was detected between TG3 antibody titers and TG2 antibodies.

Severity of small bowel mucosal enteropathy at diagnosis did not correlate with the titers of TG3 antibodies in this study, but after one year of GFD treatment there was a trend toward lower $\mathrm{Vh} / \mathrm{CrD}$ in patients with higher TG3 antibody titers initially, indicating that the mucosal damage had not recovered as well as in patients with lower TG3 antibody titers. We further detected lower intestinal $\gamma \delta+$ IEL densities both at diagnosis and on GFD in patients with high TG3 antibody titers. We have previously shown that the densities of $\gamma \delta+$ IELs are lower in DH compared to celiac disease [5], and also, $\gamma \delta+$ IELs have shown to be lower in patients with refractory celiac disease [30] and in EmA-negative celiac disease, which was associated with long-lasting, severe celiac disease in our previous study [31]. Therefore, the negative correlation between $\gamma \delta+$ IELs and TG3 antibody titers fits well to the hypothesis that TG3 antibody response is connected to advanced celiac disease. Further, it seems possible that $\gamma \delta+$ IELs might have a regulatory potential on the activity of natural killer cells [32,33], and that a lower level of this activity might result in increasing adaptive immune response and production of TG3 antibodies in addition to TG2 antibodies.

Several previous reports have shown that serum TG3 antibody titers are lower among DH patients on GFD compared to untreated patients, and we previously showed with serial patient samples that TG3 antibody titers decrease along with GFD in DH [28]. However, data about gluten-dependency of TG3 antibodies in celiac disease are scarce. To our surprise, in this study gluten-dependency of TG3 antibodies in celiac disease was not as evident as in DH. In fact, TG3 antibody titers above the cut-off level had decreased in only $56 \%$ of the patients after one year of dietary treatment. Consistently with this, it has previously been shown that serum TG6 antibodies, which have been suggested to serve as a marker identifying celiac disease patients with a risk of neurological symptoms, do not show gluten-dependency in celiac disease [34]. The reasons for divergency of TG3 and TG6 antibodies from glutendependent TG2 antibodies in celiac disease are obscure, but it might be that serum TG3 antibodies found in celiac disease are not identical with the antibodies detected in DH patients. Sardy et al. showed already in 2002 that two different types of TG3 antibody populations existed; one was directed exclusively against TG3, and the other population was primarily directed against TG2 but also cross reacted with TG3 [6]. Celiac disease patients were found to have these last-mentioned, low-avidity TG3 antibodies and DH patients high-avidity TG3 antibodies. It has also been suggested that TG3 antibodies might fluctuate with gluten intake, intestinal inflammation or trauma to the skin, and might be an indicator of disease activity only at the time of serum collection [12].

To conclude, in this study consisting of altogether 119 untreated celiac disease patients it was shown that serum TG3 antibody titers correlate with the age at celiac disease diagnosis. Further, increased levels of TG3 antibodies were connected to lower densities of small bowel mucosal $\gamma \delta+$ cells and poorer mucosal recovery on GFD. No associations were detected between TG3 antibodies and other celiac disease autoantibodies, the severity of clinical symptoms, laboratory parameters, associated diseases or celiac disease complications.

\section{Conflict of interest}

None declared.

\section{Funding}

The study was financially supported by the Finnish Medical Foundation, the Academy of Finland, the Sigrid Juselius Foundation, the Competitive State Research Financing of the Expert Responsibility area of Tampere University Hospital (Grant numbers 9P060, 9T058 and 9S020) and the Foundation for Pediatric Research.

\section{References}

[1] Dieterich W, Ehnis T, Bauer M, et al. Identification of tissue transglutaminase as the autoantigen of celiac disease. Nature Medicine 1997;3:797-801.

[2] Kim SY, Jeitner TM, Steinert PM. Transglutaminases in disease. Neurochemistry International 2002;40:85-103.

[3] Reunala T, Salmi TT, Hervonen K. Dermatitis herpetiformis: pathognomonic transglutaminase IgA deposits in the skin and excellent prognosis on a glutenfree diet. Acta Dermato-Venereologica 2015;95:917-22.

[4] Alakoski A, Salmi TT, Hervonen K, et al. Chronic gastritis in dermatitis herpetiformis: a controlled study. Clinical and Developmental Immunology 2012;2012:640630.

[5] Järvinen TT, Kaukinen K, Laurila K, et al. Intraepithelial lymphocytes in celiac disease. American Journal of Gastroenterology 2003;98:1332-7.

[6] Sàrdy M, Karpati S, Merkl B, et al. Epidermal transglutaminase (TGase 3) is the autoantigen of dermatitis herpetiformis. Journal of Experimental Medicine 2002;195:747-57.

[7] Grenard P, Bates MK, Aeschlimann D. Evolution of transglutaminase genes: identification of a transglutaminase gene cluster on human chromosome 15q15. Journal of Biological Chemistry 2001;276:33066-78.

[8] Dieterich W, Laag E, Bruckner-Tuderman L, et al. Antibodies to tissue transglutaminase as serologic markers in patients with dermatitis herpetiformis. Journal of Investigative Dermatology 1999;113:133-6.

[9] Salmi TT, Hervonen K, Laurila K, et al. Small bowel transglutaminase 2specific IgA deposits in dermatitis herpetiformis. Acta Dermato-Venereologica 2014;94:393-7.

[10] Heil PM, Volc-Platzer B, Karlhofer F, et al. Transglutaminases as diagnostically relevant autoantigens in patients with gluten sensitivity. Journal der Deutschen Dermatologischen Gesellschaft 2005;3:974-8.

[11] Marietta EV, Camilleri MJ, Castro LA, et al. Transglutaminase autoantibodies in dermatitis herpetiformis and celiac sprue. Journal of Investigative Dermatology 2008; $128: 332-5$

[12] Hull CM, Liddle M, Hansen N, et al. Elevation of IgA anti-epidermal transglutaminase antibodies in dermatitis herpetiformis. British Journal of Dermatology 2008;159:120-4.

[13] Ladinser B, Rossipal E, Pittschieler K. Endomysium antibodies in coeliac disease: an improved method. Gut 1994;35:776-8.

[14] Taavela J, Koskinen O, Huhtala H, et al. Validation of morphometric analyses of small-intestinal biopsy readouts in celiac disease. PLoS ONE 2013;8:e76163.

[15] Marsh MN, Johnson MW, Rostami K. Mucosal histopathology in celiac disease: a rebuttal of Oberhuber's sub-division of Marsh III. Gastroenterology and Hepatology From Bed to Bench 2015;8:99-109.

[16] Korponay-Szabo IR, Halttunen T, Szalai Z, et al. In vivo targeting of intestinal and extraintestinal transglutaminase 2 by coeliac autoantibodies. Gut 2004;53:641-8.

[17] Nikander R, Kannus P, Dastidar P, et al. Targeted exercises against hip fragility Osteoporosis International 2009;20:1321-8.

[18] Dimenas E, Carlsson G, Glise H, et al. Relevance of norm values as part of the documentation of quality of life instruments for use in upper gastrointestinal disease. Scandinavian Journal of Gastroenterology. Supplement 1996;221:8-13.

[19] Hallert C, Granno C, Grant C, et al. Quality of life of adult coeliac patients treated for 10 years. Scandinavian Journal of Gastroenterology 1998;33:933-8. 
[20] Svedlund J, Sjodin I, Dotevall G. GSRS - a clinical rating scale for gastrointestinal symptoms in patients with irritable bowel syndrome and peptic ulcer disease. Digestive Diseases and Sciences 1988;33:129-34.

[21] Salmi TT, Hervonen K, Kautiainen $H$, et al. Prevalence and incidence of dermatitis herpetiformis: a 40-year prospective study from Finland. British Journal of Dermatology 2011;165:354-9.

[22] Hervonen K, Karell K, Holopainen P, et al. Concordance of dermatitis herpetiformis and celiac disease in monozygous twins. Journal of Investigative Dermatology 2000;115:990-3.

[23] Salmi TT, Hervonen K, Kurppa K, et al. Coeliac disease evolving into dermatitis herpetiformis in patients adhering to normal or gluten-free diet. Scandinavian Journal of Gastroenterology 2015;50:387-92.

[24] Lohi S, Mustalahti K, Kaukinen K, et al. Increasing prevalence of coeliac disease over time. Alimentary Pharmacology and Therapeutics 2007:26:1217-25.

[25] Jaskowski TD, Hamblin T, Wilson AR, et al. IgA anti-epidermal transglutaminase antibodies in dermatitis herpetiformis and pediatric celiac disease. Journal of Investigative Dermatology 2009;129:2728-30.

[26] Lytton SD, Antiga E, Pfeiffer S, et al. Neo-epitope tissue transglutaminas autoantibodies as a biomarker of the gluten sensitive skin disease - dermatitis herpetiformis. Clinica Chimica Acta 2013;415:346-9.

[27] Borroni G, Biagi F, Ciocca O, et al. IgA anti-epidermal transglutaminase antibodies: a sensible and sensitive marker for diagnosis of DH in adult patients. Journal of the European Academy of Dermatology and Venereology 2013;27:836-41.
[28] Reunala T, Salmi TT, Hervonen K, et al. IgA anti-epidermal transglutaminase antibodies in dermatitis herpetiformis: a significant but not complete response to a gluten-free diet treatment. British Journal of Dermatology 2014:172:1139-41.

[29] Ress K, Teesalu K, Annus T, et al. Low prevalence of IgA anti-transglutaminase 1,2 , and 3 autoantibodies in children with atopic dermatitis. BMC Research Notes 2014;7:310.

[30] Koskinen O, Lindfors K, Collin P, et al. Intestinal transglutaminase 2 specific antibody deposits in non-responsive coeliac disease. Digestive and Liver Disease 2010;42:692-7.

[31] Salmi TT, Collin P, Korponay-Szabó IR, et al. Endomysial antibody-negative coeliac disease: clinical characteristics and intestinal autoantibody deposits. Gut 2006;55:1746-53.

[32] Bhagat G, Naiyer AJ, Shah JG et al. Small intestinal $\mathrm{CD}^{+}{ }^{\mathrm{TCR}}$ gammadelta ${ }^{+} \mathrm{NKG}_{2} \mathrm{~A}^{+}$intraepithelial lymphocytes have attributes of regulatory cells in patients with celiac disease. Journal of Clinical Investigation 2008; 118:281-93.

[33] Setty M, Discepolo V, Abadie V, et al. Distinct and synergistic contributions of epithelial stress and adaptive immunity to functions of intraepithelial killer cells and active celiac disease. Gastroenterology 2015;149:681-91.

[34] Lindfors $K$, Koskinen O, Laurila $K$, et al. IgA-class autoantibodies against neuronal transglutaminase, TG6 in celiac disease: no evidence for gluten dependency. Clinica Chimica Acta 2011;412:1187-90. 